\title{
A Modified Particle Filter Algorithm for Wireless Capsule Endoscope Location Tracking
}

\author{
Takahiro Ito, Daisuke Anzai and Jianqing Wang \\ Graduate School of Engineering \\ Nagoya Institute of Technology \\ Gokiso-cho, Showa-ku, Nagoya, Aichi 466-8555, Japan \\ \{anzai, wang\}@nitech.ac.jp
}

\begin{abstract}
Tracking a capsule endoscope location is one of promising application offered by implant body area networks (BANs). In this paper, we pay attention to a particle filter algorithm with received signal strength indicator (RSSI)-based localization in order to solve the capsule endoscope location tracking problem, which assumes a nonlinear transition model on the capsule endoscope location. However, the original particle filter requires to calculate the particle weight according to its condition (namely, its likelihood value), while the transition model on capsule endoscope location has some model parameters which cannot be estimated by received wireless signal. Therefore, for the purpose of applying the particle filter to the capsule endoscope tracking, this paper makes some modifications in the resampling step of the particle filter algorithm. Our computer simulation results demonstrates that the proposed tracking methods with the modified particle filter can improve the performance as compared with not only the conventional maximum likelihood (ML) localization but also the original particle filter-based location tracking.
\end{abstract}

\section{Categories and Subject Descriptors}

C.2.1 [Computer-Communication Networks]: Network Architecture and Design-Wireless communication

\section{General Terms}

Measurement

\section{INTRODUCTION}

In recent years, wireless body area networks (BANs) has attracted lots of attention with respect to medical and healthcare applications [1-3]. Wireless BANs realizes novel promising medical and healthcare applications such as wireless capsule endoscopy. In wireless capsule endoscopy systems, the location information of the capsule endoscope can help detecting diagnose gastrointestinal (GI) conditions and assisting the physicians for follow-up interventions. However,

Permission to make digital or hard copies of all or part of this work for personal or classroom use is granted without fee provided that copies are not made or distributed for profit or commercial advantage and that copies bear this notice and the full citation on the first page. To copy otherwise, to republish, to post on servers or to redistribute to lists, requires prior specific permission and/or a fee.

PFT 2013, September 30-October 02

Copyright (c) 2013 ICST 978-1-936968-89-3

DOI 10.4108/icst.bodynets.2013.253650 since a capsule endoscope moves inside a human body, nobody knows the location of the capsule endoscope in advance. Therefore, in the capsule endoscopy systems, it is significant to estimate and track the capsule endoscope location.

Various localization methods for the capsule endoscope have been so far proposed [4-9]. For example, localization technologies include ultrasound [4], time of arrival (TOA)-based pattern recognition [5], magnetic tracking [6,7] and computer vision $[8,9]$. In these technologies, the localization with radio frequency $(\mathrm{RF})$ signals are advantageous in terms of relatively low cost for implementation due to requiring no specific device. In this paper, we pay attention to a received signal strength indicator (RSSI)-based location estimation method. This is because it is advantageous in terms of cost and energy consumption since most of the current wireless communication standards have a function of measuring RSSI in their protocols. As related works, a RSSI-based triangulation system, which employs an external sensor array which measures signal strength of capsule transmissions at multiple points, has been proposed, and the average experimental error is reported to be $37.7 \mathrm{~mm}$ [10]. In order to further improve the performance of RSSI-based localization, the use of a maximum likelihood (ML) estimation is effective $[11,12]$. However, to use an ML estimation method, a statistical model on the RSSI, which can well characterize the real variation of the RSSI in the location estimation area is required in advance. So, in order to successfully apply an ML estimation technique to capsule endoscope localization, we have so far investigated the statistical model of the propagation characteristics of medical implant communication services (MICS) band signals by using finite-difference timedomain (FDTD) simulations together with an anatomical human body model. [13].

In applying the RSSI-based ML localization to a capsule endoscope location tracking problem, it is important to take its behavioral nature in consideration because the capsule endoscope is continuously localized. For example, its present location is strongly related to its past locations, so we can track it better by low-pass filtering its past locations. Furthermore, if we know the mathematical model of the location transition of the capsule endoscope, the tracking performance can be improved even more. However, in the case of a capsule endoscope tracking, the model is expected to be nonlinear in reality, so we can have two approaches in this case, one is an extended Kalman filter and the other is a par- 
ticle filter [14-17]. In this paper, we focus on the particle filter-based tracking method. As for the transition model of the capsule endoscope location, the movement can be well modeled as a random way point model [18]. If we employ the random way point model as the transition model of the capsule endoscope, some parameters in the model can not be estimated by received wireless signal. The original particle filter requires to calculate the particle weight according to its condition (namely, its likelihood value), so it is difficult to directly apply the particle filter to the capsule endoscope location tracking. Therefore, in order to solve this problem, we propose to modify the resampling step of the particle filter algorithm.

In this paper, to begin with, we present the propagation characteristics of the $400 \mathrm{MHz}$ MICS band signals, and explain the ML localization method based on the propagation characteristics. Then, Section 3 proposes the modified particle filter-based tracking method to successfully deal with the random way point state transition model. Finally, in Section 4 , we evaluate the performances of the modified particle filter algorithm for location tracking by computer simulations, and discuss the location estimation accuracies in the capsule endoscopy systems.

\section{CONVENTIONAL ML LOCALIZATION}

\subsection{System Model}

We assume that a capsule endoscope is located inside a human body and $M$ receivers are put onto the human body as shown in Fig. 1. The positions of receivers are known in advance and the capsule endoscope location is unknown, so the capsule endoscope location should be estimated by the known receiver location information. We define the threedimensional locations of the capsule endoscope and the $m$-th receiver $(m=1, \cdots, M)$ as

$$
\begin{aligned}
\mathbf{r} & =[x, y, z]^{T} \\
\mathbf{r}_{m} & =\left[x_{m}, y_{m}, z_{m}\right]^{T}
\end{aligned}
$$

where $(\cdot)^{T}$ indicates the transpose of $(\cdot)$.

\subsection{Propagation Characteristics}

To introduce an ML estimation technique into the RSSIbased localization, we have employed an FDTD simulation with an anatomically-based numerical human body model to investigate the propagation characteristics of implant BAN signals [13]. From our investigation based on the FDTD simulations, we have found that the variation of the RSSIs can be well expressed with the following two-layered model:

$$
\begin{aligned}
\mu(d) & =\log \overline{P_{r}}=\log \left[\alpha d^{-n}\right] \\
p\left(P_{r} \mid d\right) & =\frac{1}{\sqrt{2 \pi \sigma P_{r}}} \exp \left[-\frac{\left\{\log P_{r}-\mu(d)\right\}^{2}}{2 \sigma^{2}}\right] .
\end{aligned}
$$

Here, Eq.(3) means the average received power $\overline{P_{r}}$ against the transceiver distance $d$, and Eq.(4) indicates that the conditional probability density function on RSSI $P_{r}$ can be characterized by the Log-Normal distribution when the transceiver distance $d$ is given. Note that the channel model parameters in Eqs.(3) and (4) have been obtained by the FDTDsimulated data as $\alpha=10^{-7.50}, n=6.65$ and $\sigma=5.50 \mathrm{~dB}$ in [13].
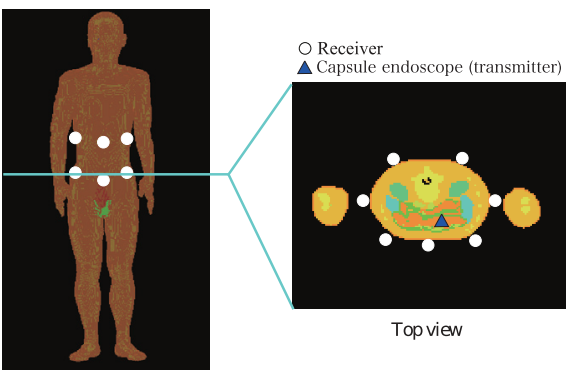

Front view

Figure 1: System model for capsule endoscope localization

\subsection{Localization}

The log-likelihood function on $\mathbf{r}$ is defined as

$$
L(\mathbf{r})=\log p(\mathbf{P} \mid \mathbf{r})
$$

where $\mathbf{P}$ is a measured RSSI vector $\mathbf{P}=\left[P_{1}, \cdots, P_{M}\right]^{T}$. Based on the investigation of the propagation characteristics, replacing $d$ by $d_{m}(\mathbf{r})$ and $P_{r}$ by $P_{m}$ respectively in (3) and (4) (also $\overline{P_{r}}$ by $\bar{P}$ ), Eq. (5) can be re-written as:

$$
\begin{aligned}
L(\mathbf{r}) & =\log \left[\prod_{m=1}^{M} p\left(P_{m} \mid d_{m}(\mathbf{r})\right)\right] \\
& =\sum_{m=1}^{M}\left[-\log \sqrt{2 \pi} \sigma P_{m}-\frac{\left(\log P_{m}-\mu\left[d_{m}(\mathbf{r})\right]\right)^{2}}{2 \sigma^{2}}\right](6)
\end{aligned}
$$

where $d_{m}(\mathbf{r})$ denotes the distance between the capsule endoscope and the $m$-th receiver. The ML Localization method gives the estimated location $\hat{\mathbf{r}}_{M L}$ as a value of $\mathbf{r}$ which maximizes $L(\mathbf{r})$, that is, $\hat{\mathbf{r}}_{M L}$ can be obtained from the following maximum likelihood equation:

$$
\left.\frac{\partial L(\mathbf{r})}{\partial \mathbf{r}}\right|_{\mathbf{r}=\hat{\mathbf{r}}_{M L}}=0 .
$$

\section{MODIFIED PARTICLE FILTER ALGO- RITHM FOR LOCATION TRACKING}

Particle filter is widely known as a sequential Monte Carlo method, which is used as one of tools for Bayesian inference. The probability densities can be represented by point mass (of "particle") and the particles have information of the parameters of the state transition model. As mentioned below, the particle weight represents the likelihood value only on the particle location, so the effect for some parameters of the state transition model can not be considered in the update step. To successfully apply the particle filter to the capsule endoscope location tracking problem, we modify the resampling step in the particle filter algorithm to take care of all the state transition model parameters. The particle filter algorithm repeats the following four steps in an iterative manner.

\subsection{Prediction Step}

The state of each particle is determined by state transition model of the capsule endoscope location. This paper assumes the following random way point model as the realistic 
transition model [18]:

$$
\begin{aligned}
\mathbf{r}_{i}[n] & =\mathbf{r}_{i}[n-1]+v_{i}[n] \mathbf{e}_{i}^{k} \Delta t \\
v_{i}[n] & \sim \mathcal{N}\left(\bar{v}, \sigma_{v}\right) \\
\mathbf{e}_{i}^{k} & =\frac{\mathbf{g}_{i}^{k}-\mathbf{g}_{i}^{k-1}}{\left|\mathbf{g}_{i}^{k}-\mathbf{g}_{i}^{k-1}\right|} \\
\mathbf{g}_{i}^{k} & \sim\left[\begin{array}{l}
\mathcal{U}(0, L X) \\
\mathcal{U}(0, L Y) \\
\mathcal{U}(0, L Z)
\end{array}\right]
\end{aligned}
$$

where $\mathbf{r}_{i}[n], v_{i}[n], \bar{v}, \sigma_{v}, \Delta t$ and $\mathbf{g}_{i}^{k}$ represent the $i$-th particle location at the discrete-time index of $n$, the velocity of the $i$-th particle at the discrete-time index of $n$, the average velocity of the capsule endoscope moving, the standard deviation of the capsule endoscope velocity, the time interval of the location tracking and the $k$-th destination location vector of the $i$-th particle, respectively, and $\mathcal{N}(\mu, \sigma)$ and $\mathcal{U}(a, b)$ are the Gaussian distribution with mean $\mu$ and standard deviation $\sigma$, and the Uniform distribution between $a$ and $b$, respectively. Note that once particle reaches the destination location (that is $\mathbf{g}^{k}=\mathbf{r}_{n}$ ), $k$ becomes $k+1$ so that $\mathbf{g}^{k}$ and $\mathbf{e}^{k}$ will be updated.

\subsection{Update Step}

In the update step, the weight of each particle $\tilde{w}_{i}[n]$ at the time index of $n$ is updated as in the following way:

$$
\begin{aligned}
\tilde{w}_{i}[n] & =w_{i}[n-1] p\left(\mathbf{P}_{1, i}, \mathbf{P}_{2, i}, \cdots, \mathbf{P}_{M, i} \mid \mathbf{r}_{i}[n]\right) \\
& =w_{i}[n-1] \prod_{m=1}^{M} p\left(\mathbf{P}_{m, i} \mid \mathbf{r}_{i}[n]\right) \\
w_{i}[n] & =\frac{\tilde{w}_{i}[n]}{\sum_{j=1}^{N_{p}} w_{j}[n]}
\end{aligned}
$$

where $w_{i}[n], N_{p}$ is the normalized weight of the $i$-th particle, the number of particles, respctively. In Eq.(6), the particle weight $\tilde{w}_{i}[n]$ can be calculated by the likelihood function represented in Eq.(6).

\subsection{Resampling Step with Modification for In- troducing Random Way Point State Tran- sition Model}

As the particle filter algorithm is iteratively performed, some normalized particle weights become negligible. This leads to degeneration of the particles, and finally, this phenomenon gives a quite bad influence on the sequential Monte Carlo method. From this reason, it is necessary to remove the particles whose weights are relatively small and regenerate them. For this purpose, we define the effective parameter $\beta_{e f f}$ as

$$
\beta_{e f f}=\frac{1}{\sum_{i=1}^{N_{p}} w_{i}^{2}[n]} .
$$

Note that $\beta_{\text {eff }}$ ranges from $1 / N_{p}$ to 1 . Resampling will be proceeded if $\beta_{\text {eff }}<\beta_{t h r}$, where $\beta_{t h r}$ is a threshold which indicates the particles degeneration. Some particles with negligible weights are eliminated and regenerated as the copy of the particle with a relatively large weight. Here, the original particle is chosen with the probability according to its weight. All the particle weights are then set to $1 / N_{p}$. However, we herein point out that the particle weight is evalu-
Table 1: Parameters of computer simulations

\begin{tabular}{|c|c|}
\hline$L X$ & $40 \mathrm{~cm}$ \\
\hline$L Y$ & $40 \mathrm{~cm}$ \\
\hline$L Z$ & $20 \mathrm{~cm}$ \\
\hline Number of particles $N_{p}$ & 1000 \\
\hline Resampling threshold $\beta_{t h r}$ & 0.6 \\
\hline$\alpha$ & $10^{-7.50}$ \\
\hline$n$ & 6.65 \\
\hline$\sigma$ & $5.50 \mathrm{~dB}$ \\
\hline$\Delta t$ & $0.1 \mathrm{~s}$ \\
\hline $\bar{v}$ & $0.05 \mathrm{~cm} / \mathrm{s}$ \\
\hline$\sigma_{v}^{2}$ & $0.005(\mathrm{~cm} / \mathrm{s})^{2}$ \\
\hline
\end{tabular}

ated based only on the likelihood value on its particle location, that is, the other parameter, for example, the destination location of the particle, does not reflect its weight. In order to consider the effect of this parameter, we make the following additional procedure in the resampling step:

$$
\begin{aligned}
\mathbf{g}_{i}^{k, \text { modifed }} & =\mathbf{g}_{i}^{k, \text { original }}+\mathbf{g}_{i}^{\text {pos }} \\
\mathbf{g}_{i}^{\text {pos }} & \sim \mathcal{N}\left(0, \sigma_{\text {pos }}\right)
\end{aligned}
$$

where $\mathbf{g}_{i}^{k, \text { modified }}, \mathbf{g}_{i}^{k, \text { original }}$ and $\sigma_{\text {pos }}$ denote the modified destination location vector of the $i$-th particle, the destination location vector of the $i$-th particle regenerated in the original particle filter algorithm and the standard deviation of variational factor $\mathbf{g}_{i}^{\text {pos }}$, respectively.

\subsection{Estimation Step}

Finally, the estimated location of the particle filter is calculated as

$$
\hat{\mathbf{r}}_{P F}[n]=\sum_{i=1}^{N_{p}} w_{i}[n] \mathbf{r}_{i}[n] .
$$

\section{PERFORMANCE EVALUATION 4.1 Simulation Environment}

In order to evaluate the performances of the proposed location tracking method, we performed computer simulations. In the computer simulations, we assumed that the capsule endoscope moves inside a small intestine according to the random way point model described in the previous section, and the destination location of the transition model was determined based on the small intestine of the numerical human model in advance as shown in Fig. 2. The estimation area was assumed to a cuboid area around the small intestine, whose size was $L X=40 \mathrm{~cm}$ (width) $\times L Y=40 \mathrm{~cm}$ (height) $\times L Z=20 \mathrm{~cm}$ (depth). We put 8 receivers at each vertex of the cuboid. The computer simulation parameters are summarized in Table 1.

\subsection{Results}

Fig. 3 shows the root mean square (RMS) location estimation error for the modified particle filter algorithm against the standard deviation $\sigma_{\text {pos }}$ in the resampling step. As can be seen from Fig. 3, the location estimation performance is insensitive to both the resampling threshold $\beta_{t h r}$ and $\sigma_{\text {pos }}$. Since the resampling threshold $\beta_{t h r}$ has a trade-off relationship between the location estimation accuracy and the convergence performance, we use $\sigma_{p o s}=7 \mathrm{~cm}$ and $\beta_{t h r}=0.6$ 


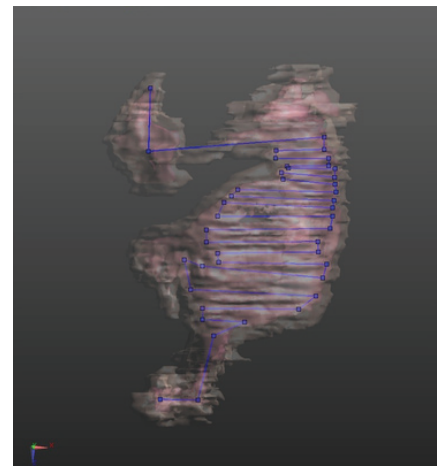

Figure 2: trajectory of capsule endoscope movement

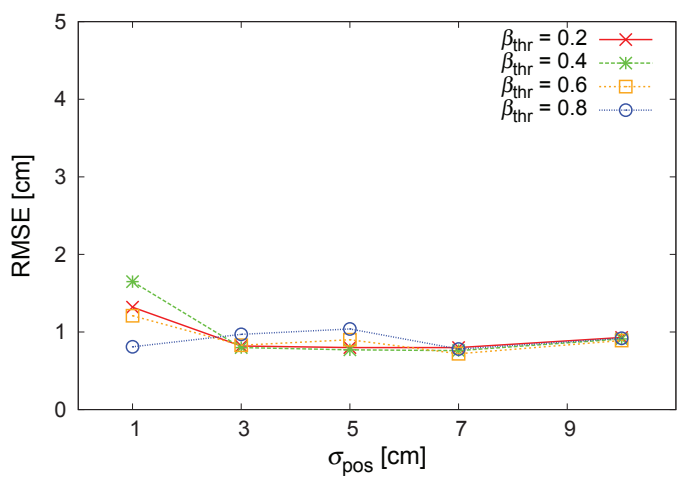

Figure 3: Effect of $\sigma_{p o s}$ and resampling threshold $\beta_{t h r}$ on the particle filter-based location tracking

for the quick convergence and optimal localization performance.

Fig. 4 shows the RMS location estimation error for the proposed and original particle filter algorithms. Furthermore, Fig.4 also includes the localization performance for the conventional ML localization and the theoretical bounds for the ML localization, namely, Cramer-Rao lower bound (CRLB) [19]. As can be seen from this figure, the performance of the ML localization converges to the CRLB, therefore, the efficiency of the ML estimator can be established. On the other hands, Fig. 4 also shows the results for the original particle filter algorithm with true destination vector $\mathbf{g}_{i}^{k}$ information, which can be realized only in a computer simulation, that is, it cannot be realized in a reality because no one can obtain the true destination of the capsule endoscope. From these results, the performance of the original particle filter does not converge because the particle weight cannot represent the destination of the capsule endoscope, whereas the proposed algorithm significantly improves the location estimation performance, and furthermore, the localization performance of the modified particle algorithm converges to a good estimation accuracy. Moreover, the proposed algorithm accomplishes the estimation accuracy for the original particle filter algorithm with true destination information. Therefore, it can be concluded that the proposed particle filter algorithm properly

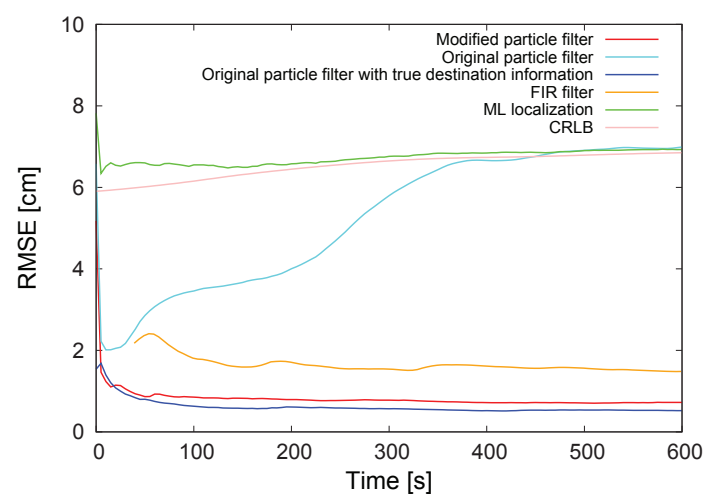

Figure 4: Comparison of location estimation accuracies between FIR filter- and particle filter-based tracking methods

deals with the destination information of each particle.

Then, let us compare the performances between the proposed particle filter algorithm and another filter algorithm for wireless capsule endoscope tracking. As comparison purpose, we choose a finite impulse response (FIR) filter algorithm, which tracks the capsule endoscope location by averaging its past locations [20]. Fig. 4 also includes the localization performance of the FIR filter algorithm. As compared with the two location tracking methods, we have confirmed that the localization accuracy for the modified particle filter algorithm is better than that for the FIR filter algorithm. This reason is that the proposed particle filter can successfully make use of the realistic capsule endoscope transition model. For example, the achievable RMS location estimation errors for the FIR filter and the proposed particle filter algorithms are $1.5 \mathrm{~cm}$ and $0.7 \mathrm{~cm}$, respectively, so the proposed particle filter algorithm can improve the performance by around $50 \%$ as compared with the FIR filter algorithm.

Finally, Fig.5 shows the cumulative distribution functions $(c d f \mathrm{~s})$ on the location estimation error for the particle filter based- and the FIR filter based-location tracking methods and the ML localization. Similarly to the RMS location estimation error, the proposed particle filter algorithm outperforms the original particle filter and the FIR filter algorithms in term of the $c d f$ on the location estimation error. From Fig.5, the maximum location estimation error of the proposed article filter algorithm is achieved within $2 \mathrm{~cm}$, whereas that of the FIR filter is around $2.5 \mathrm{~cm}$, and when the $c d f$ of the location estimation error is 0.7 , the proposed particle filter algorithm improves the estimation performance by around $50 \%$ as compared with the FIR filter algorithm.

\section{CONCLUSIONS}

This paper has introduced the particle filter algorithm into the RSSI-based localization for wireless capsule endoscope. In order to successfully apply the particle filter algorithm to the capsule endoscope tracking problem, we have proposed to modify the resampling step in the particle filter algorithm because the transition model has some param- 


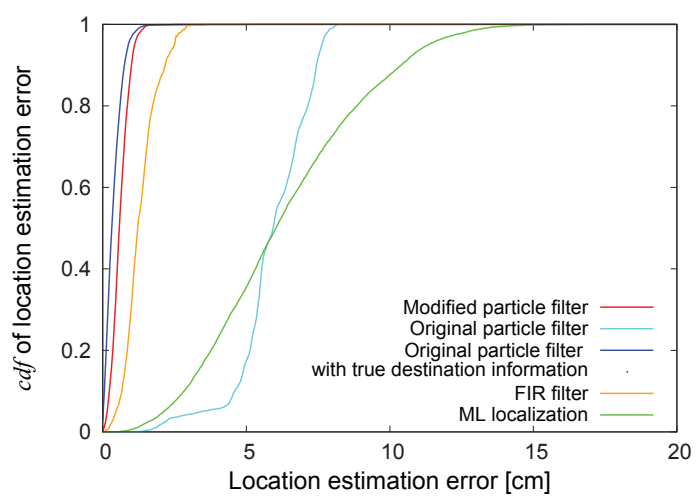

Figure 5: $c d f$ of location estimation error

eters that cannot be estimated by received wireless signal. Our computer simulation results have demonstrated that the particle particle filter algorithm outperforms not only the original particle filter algorithm but also the conventional FIR filter algorithm due to taking the realistic capsule endoscope transition model into consideration. We would like to remark that our developed location tracking method can accomplish a high estimation accuracy of $0.7 \mathrm{~cm}$.

Our future subject is to introduce the particle filter-based tracking into other localization method, such as TOA-based localization to further improve the estimation accuracy of the capsule endoscope location.

\section{Acknowledgment}

This study was supported partially by JSPS KAKENHI Grant Number 24760297 and JST Adaptable and Seamless Technology transfer Program (A-STEP).

\section{REFERENCES}

[1] H.B. Li and R. Kohno, "Body area network and its standardization at IEEE 802.15. BAN," Advances in Mobile and Wireless Communications, pp. 223-238, Springer, 2008.

[2] E. Monton, J.F. Hernandez, J.M. Blasco, T. Herve, J. Micallef, I. Grech, A. Brincat, and V. Traver, "Body area network for wireless patient monitoring," IET Communications, vol. 2, no. 2, pp. 215-222, Feb. 2008.

[3] J. Wang and Q. Wang, Body Area Communications, Wiley-IEEE, 2013.

[4] K. Arshak and F. Adepoju, "Capsule tracking in the GI tract: a novel microcontroller based solution," in Proc. IEEE Sensors Applications Symposium, 2006, pp. 186-191.

[5] M. Kawasaki and R. Kohno, "A TOA based positioning technipue of medical implanted devices," in Third International Symposium on Medical Information \&S Communication Technology, ISMICT09, Monteral, 2009.

[6] X. Wang, M. Meng, and C. Hu, "A localizatio method using 3-axis magnetoresistive sensors for tracking of capsule endoscope," in Proc. of IEEE/EMBS, Soul, 2006, pp. 2522-2525.
[7] C.Hu, M. Meng, and M. Mandal, "The calibration of 3-axis magnetic sensor array system for tracking wireless capsule endoscope," in IEEE/RSJ International Conference on Robots and Systems, 2006, pp. 162-167.

[8] J. Bulat, K. Duda, M. Duplaga, R.Fraczek, A.Skalski, M. Socha, P.Turcza, and T. Zielinski, "Data processing tasks in wireless GI endoscopy: image-based capsule localization and navigation with video compression," in Proc. of IEEE/EMBS, 2007, pp. 2815-2818.

[9] R. Kuth, J. Reinschke, and R. Rockelein, "Method for determining the position and orientation of an endoscopy capsule guided through an examination object by using a navigating magnetic field generated by means of a navigation device," Patent US2007/0 038 063, February 15, 2007.

[10] M.Fischer, R.Schreiber, D.Levi, and R.Eliakim, "Capsule endoscopy: the localization system," Gastrointestinal endoscopy clinics of north america, vol. 14, pp. 25-31, 2004.

[11] D. Anzai and S. Hara, "A simple outlier data rejection algorithm for an RSSI-based ML location estimation in wireless sensor networks," in Proc. IEEE VTC 2008-Fall, pp. 1-5, Sept. 2008.

[12] R. Zemek, D. Anzai, S. Hara, K. Yanagihara, and K. Kitayama, "RSSI-based localization without a prior knowledge of channel model parameters," International Journal of Wireless Information Networks, vol. 15, no. 3-4, pp. 128-136, Oct. 2008.

[13] D. Anzai, S. Aoyama, J. Wang, "Performance evaluation on RSSI-based localization for capsule endoscopy systems with $400 \mathrm{MHz}$ MICS band signals," IEICE trans. commun., vol. E95-B, no. 10, pp. 3081-3087, Jan. 2012.

[14] R.B. Noland, W.Y. Ochieng, and M. Quddus, "An extended Kalman filter algorithm for integrating GPS and low cost dead reckoning system data for vehicle performance and emissions monitoring," Journal of Navigation, vol. 56, no. 2, pp. 257-275, 2003.

[15] F.A. Faruqi and K.J. Turner, "Extended Kalman filter synthesis for integrated global positioning/inertial navigation systems," Applied Mathematics and Computation, vol. 115, no. 2-3, pp. 213-227, 2000.

[16] B. Ristic, S. Arulampalam, and N. Gordon, Beynod the Kalman Filter - Particle filters for tracking applications. Artech House, 2004.

[17] M. S. Arulampalam, S. Maskell, N. Gordon, and T. Clapp, "A tutorial on particle filters for online nonliner/nongaussian bayesian tracking," IEEE Trans. on Signal Pro- cessing, vol. 50, pp. 174-188, Feb. 2002.

[18] B. Moussakhani, R. Chavez-Santiago, I. Balasingham, "Multi model tracking for localization in wireless capsule endoscopes," ISABEL '11 Proceedings of the 4 th International Symposium on Applied Sciences in Biomedical and Communication Technologies Article No. 159

[19] H. L. Van Trees, Detection, Estimation, and Modulation Theory: Part I, WILEY, 1968.

[20] S.O. Haykin, Adaptive Filter Theory, Prentice Hall, 2001. 